\title{
Free Vascularized Fibular Grafting Improves Vascularity Compared With Core Decompression in Femoral Head Osteonecrosis: A Randomized Clinical Trial
}

\author{
Lu Cao MD, Changan Guo MD, Jifei Chen MD, Zenggan Chen MD, \\ Zuoqin Yan MD
}

Received: 13 January 2017/ Accepted: 26 April 2017/Published online: 4 May 2017

(C) The Association of Bone and Joint Surgeons (B) 2017

\begin{abstract}
Background Management of osteonecrosis of the femoral head remains challenging. Core decompression and free vascularized fibular grafting are commonly used surgical procedures for treatment of osteonecrosis of the femoral head. Few studies, however, have compared these two procedures in a randomized controlled study, in terms of improved vascularity of the femoral head, progression of disease, or hip scores.

Question/purposes (1) What is the effect of core decompression and fibular grafting on vascularity of the femoral head as measured by single-photon emission CT (SPECT)/CT? (2) Does one of these two methods lead to greater progression of Association Research Circulation Osseous (ARCO) stage as determined by serial MRI? (3) What is the relationship between the change in vascularity
\end{abstract}

Funding for this study was received from the Natural Science Foundation of China (81672157, ZY; 81401820, LC) and the Major Project and Disease of the Shanghai Health System (2014ZYJB0302; ZY) from the Shanghai Municipal Commission of Health and Family Planning.

All ICMJE Conflict of Interest Forms for authors and Clinical Orthopaedics and Related Research ${ }^{\circledR}$ editors and board members are on file with the publication and can be viewed on request.

Clinical Orthopaedics and Related Research ${ }^{\mathbb{R}}$ neither advocates nor endorses the use of any treatment, drug, or device. Readers are encouraged to always seek additional information, including FDAapproval status, of any drug or device prior to clinical use.

Each author certifies that his institution approved or waived approval for the human protocol for this investigation and that all investigations were conducted in conformity with ethical principles of research

L. Cao, C. Guo, J. Chen, Z. Chen, Z. Yan ( $\square)$

Department of Orthopedic Surgery, Zhongshan Hospital, Fudan

University, No. 180 Fenglin Road, Shanghai 200032, China

e-mail: yan.zuoqin@zs-hospital.sh.cn; 18616880818@163.com of the femoral head and hip function as measured by the Harris hip score (HHS) and progression to THA as an endpoint?

Methods A randomized controlled trial was performed between June 2010 and October 2012 at Zhongshan Hospital, Fudan University. During the study period, 51 patients who presented with ARCO Stages I to IIIB bilateral osteonecrosis were potentially eligible for inclusion, and 33 patients were identified as meeting the inclusion criteria and offered enrollment and randomization. Six patients declined to participate at the time of randomization, leaving a final sample of 27 participants (54 hips). Bilateral hips of each patient were randomly assigned to surgical options: one side was treated with core decompression and the contralateral side was concurrently treated with fibular grafting. SPECT/CT examinations were performed to quantify radionuclide uptake to evaluate vascularity of the femoral head before treatment and at 6 and 36 months after surgery. With the numbers available, we found no differences between the groups regarding vascularity at baseline $(64 \% \pm 8 \%$ core decompression-treated hips versus $64 \% \pm 7 \%$ in the fibular-grafted hips; $95 \% \mathrm{CI},-5 \%$ to $5 \%$; $\mathrm{p}=0.90$ ). MR images of the hips were obtained before surgery and at $6,12,24$, and 36 months postoperatively and staged based on the ARCO classification. All patients were assessed clinically before treatment and followed up at $6,12,18,24,30$, and 36 months after treatment using the HHS. We considered a difference in the HHS of 10 as the minimal clinically important difference (MCID). Patient progression to THA was defined as the endpoint for followup. Six patients (22\%) were lost to followup.

Results By SPECT/CT analysis, decompression-treated hips had lower vascularity than fibular-grafted hips at 6 months $(68 \% \pm 6 \%$ versus $95 \% \pm 5 \%$; mean difference, $-27 \%$; $95 \% \mathrm{CI},-32 \%$ to $-23 \% ; \mathrm{p}<0.001)$ and 36 months $(57 \% \pm 4 \%$ versus $91 \% \pm 3 \%$; mean difference, 
$-34 \%$; $95 \% \mathrm{CI},-37 \%$ to $-32 \%$; $\mathrm{p}<0.001)$. MRI analysis showed no differences between decompression-treated hips and fibular-grafted hips regarding ARCO stage at 12 months $(p=0.306)$ and 24 months $(p=0.06)$. Progression of ARCO staging was more severe in the decompression group than the fibular grafting group at 36 months $(\mathrm{p}=$ 0.027). The mean HHS was lower in the decompression group than in the fibular grafting group throughout the followup period, although these differences were at or below the MCID of 10 points early on. However, by 18 months, the scores favored fibular grafting ( $72 \pm 4$ versus $84 \pm 4$; mean difference, $-13 ; 95 \%$ CI, -15 to -7 ; $<<$ 0.001 ), a finding that was maintained at 24,30 , and 36 months. We found no differences between decompressiontreated hips and fibular-grafted hips regarding progression to THA at 36 months (two of $21 ; p=0.893$ ).

Conclusions Hips that underwent a vascularized fibular grafting procedure fared better than hips receiving core decompression as measured by improved vascularity and less progression of osteonecrosis as measured by ARCO staging. The mean HHS of the fibular-grafted hips was better than that of the decompression-treated hips during the entire postoperative period, but the differences were modest early on, and for the early postoperative period the differences were unlikely to have been clinically important; by 18 months after surgery, the differences probably were clinically important. The mid-term outcomes associated with vascularized fibular grafting seen in our patients are associated with improvements in femoral head vascularity and the potential for bone revitalization.

Level of Evidence Level I, therapeutic study.

\section{Introduction}

Osteonecrosis of the femoral head is a relatively common condition that can be especially devastating because of its propensity to affect young individuals $[18,24,29]$. It is usually bilateral [27], and management is challenging. Although the pathogenesis of osteonecrosis remains poorly understood, it is generally accepted that various traumatic and nontraumatic insults compromise the already precarious circulation of the femoral head, leading to bone marrow and osteocyte death [17]. Without treatment, more than $70 \%$ of femoral heads with osteonecrosis collapse and require prosthetic replacement within 3 years of diagnosis according to Ohzono et al. [20], who observed the natural progression of 115 untreated hips. Early intervention before collapse may improve the success of joint-preserving procedures.

Numerous treatment options have been described to prevent or at least delay disease progression and femoral head collapse [11, 21]. Core decompression is a surgical procedure that can reduce intramedullary pressure, one of the main pathogenic mechanisms of avascular necrosis, particularly in precollapse stages [15]. Creeping substitution to the necrotic area occurs by bringing a blood supply through drilled channels after decreasing the intramedullary pressure [23, 25]. Bednarek et al. [4] investigated 63 patients with osteonecrosis (72 hips) who were treated with core decompression filled with bone grafts and reported that 45 hips (63\%) obtained pain relief with preservation of a spherical femoral head after 1 year followup. In recent years, modified core decompression has become one of the most-reliable and most-commonly used methods for treating the early stages of osteonecrosis of the femoral head $[4,9,11,27,34]$.

Free vascularized fibular grafting is another important hip-preserving approach for treatment of osteonecrosis. A vascularized fibula provides support for the articular surface, reduces intraosseous pressure, removes and replaces the necrotic tissue, and improves the biologic microenvironment of the region [3, 28, 31]. Ünal et al. [28] reported that free vascularized fibular grafting yielded excellent postoperative Harris hip scores (HHS) in 13 patients (50\%) and good HHS in eight patients (21\%) in a 7.6-year followup study. It is believed that the key role of this microsurgical technique is to provide adequate revascularization to the new subchondral bone while at the same time maintaining appropriate mechanical support of the femoral head $[6,7,13]$.

Neither core decompression nor free vascularized fibular grafting are new. However, to our knowledge, no published study has compared the effect of decompression and fibular grafting on the blood supply of the femoral head or the relationship between blood supply and hip function. Therefore, we conducted a randomized controlled clinical trial in which both surgical procedures were used to treat patients with bilateral osteonecrosis. We asked the following questions: (1) What is the effect of core decompression and fibular grafting on vascularity of the femoral head as measured by single-photon emission CT (SPECT)/CT? (2) Does one of these two methods lead to greater progression of Association Research Circulation Osseous (ARCO) stage as determined by serial MRI? (3) What is the relationship between the change in vascularity of the femoral head and hip function as measured by the HHS and progression to THA as an endpoint?

\section{Patients and Methods}

This study was a randomized, controlled, single-institution trial of two surgical procedures performed in the same patients for treatment of bilateral osteonecrosis of the 
Fig. 1 The flow chart shows distribution of the study subjects from enrollment to the end of the study.

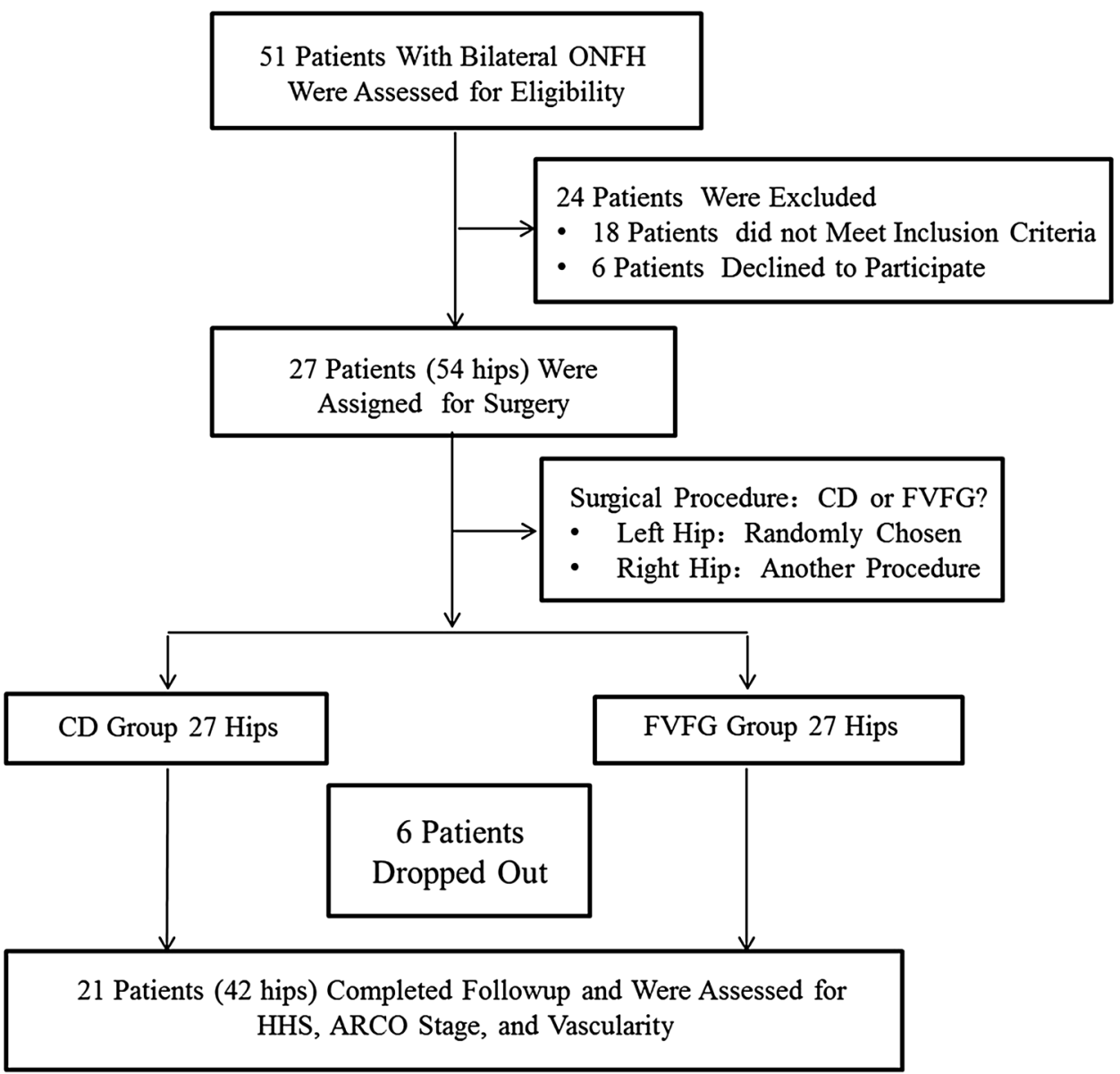

femoral head. From June 2010 to October 2012, patients with bilateral osteonecrosis were recruited at the Zhongshan Hospital of Fudan University. The diagnosis and staging of bilateral osteonecrosis were made based on plain radiographs and MR images according to the ARCO classification system [19]. The inclusion criteria for patients consisted of age between 18 and 55 years and the presence of ARCO Stages I to IIIB bilateral osteonecrosis. The exclusion criteria consisted of pregnancy; a history of femoral head and neck fracture, tumor, or any other hip disorder; current or previous infections; previous surgical or invasive intervention on a hip, including core decompression, bone graft implantation, titanium implantation, or osteotomy; steroid treatment during the last 6 months; diagnosis of a systemic disorder such as diabetes, rheumatoid arthritis, or hepatitis; inability to understand or follow instructions; and mental health problems.

Twenty-seven patients (54 hips) with osteonecrosis were enrolled in the current study. These patients were randomly assigned to receive a core decompression augmented with autologous bone grafting in one hip and concurrent contralateral free vascularized fibular grafting. For each patient, surgery was first performed on the left hip, and the selection of decompression or fibular grafting was made following the randomization sequence created by a third party (TF) not involved in this study; the other surgical procedure was performed on the right hip under the same anesthetic.

Followups were done at $6,12,18,24,30$, and 36 months postoperatively. Six patients $(22 \%)$ did not complete followup, including five who were excluded from the vascularity assessment owing to lack of a SPECT/CT examination. A total of 21 patients (42 hips) completed the entire 3-year followup and were assessed for vascularity, HHS, and ARCO stage (Fig. 1).

The etiology of the disease was believed related to steroid medication in seven patients, alcohol abuse in eight, and idiopathic in six (Table 1). According to the ARCO classification, three hips in the core decompression group were classified as Stage I, thirteen as Stage II, and five as Stage III; two hips in the fibular grafting group were classified as Stage I, thirteen as Stage II, and six as Stage III. There were 12 instances of patients with a different ARCO classification in each hip. With the numbers available, we found no differences between groups regarding quantitative SPECT/CT vascularity at baseline (core 
Table 1. Baseline characteristics of study participants

\begin{tabular}{ll}
\hline Characteristics & 21 patients (42 hips) \\
\hline Age (years; mean \pm SD [range]) & $31 \pm 6(21-48)$ \\
Sex (female/male) & $5 / 16$ \\
Risk factors & \\
Corticosteroid use & 7 \\
Alcohol abuse & 8 \\
Idiopathic & 6 \\
\hline
\end{tabular}

Table 2. ARCO stage, vascularity, and HHS of CD-treated hips and FVFG-treated hips at baseline

\begin{tabular}{lccc}
\hline \multicolumn{3}{c}{ ARCO stage Core decompression Free vascularized fibular -treated } \\
\multicolumn{4}{r}{ hips grafted-treated hips p Value* } \\
I & $3 / 21$ & $2 / 21$ & 0.631 \\
II & $13 / 21$ & $13 / 21$ & \\
III & $5 / 21$ & $6 / 21$ & \\
Vascularity (\%) & $64 \pm 8$ & $64 \pm 7$ & 0.90 \\
HHS & $61 \pm 7$ & $59 \pm 9$ & 0.39
\end{tabular}

*p values correspond to intergroup differences based on the paired $\mathrm{t}$ test (for continuous data) and Wilcoxon (for nonparametric data); ARCO = Association Research Circulation Osseous; HHS = Harris hip score; $\mathrm{CD}=$ core decompression $; \mathrm{FVFG}=$ free vascularized fibular grafting.

decompression-treated hips: $64 \% \pm 8 \%$ versus fibulargrafted hips: $64 \% \pm 7 \%$; mean difference, $0.31 \%$; $95 \% \mathrm{CI}$, $-5 \%$ to $5 \% ; \mathrm{p}=0.90$ ) (Table 2). We found no differences between groups regarding HHS at baseline (decompression-treated hips: $61 \pm 7$ versus fibular-grafted hips $59 \pm$ 9; mean difference, 2; 95\% CI, -3 to $7 ; \mathrm{p}=0.39$ ) (Table 2). We also observed no association $(\mathrm{p}=0.631)$ between the two groups in terms of baseline ARCO stages (Table 2).

\section{Surgical Procedure}

\section{Core Decompression and Bone Grafting}

Surgery was performed with the patients under general anesthesia and in the supine position. A 2- to 3-cm straight incision was made from the tip of the greater trochanter distally. A 2.5-mm Kirschner pin was inserted toward the anterolateral necrotic area of the femoral head until it was $0.5 \mathrm{~cm}$ beneath the subchondral bone, as observed with the C-arm radiograph. A cannulated custom-made hollow reamer then was used to broach the tunnel along the pin to $0.5 \mathrm{~cm}$ below the subchondral bone; part of the necrotic bone was removed from the reamer, and a curette was used to remove the rest of the necrotic bone. We designed a different diameter $\mathrm{T}$-shaped hand tap to broach the tunnel to 10 to $15 \mathrm{~mm}$, which was determined based on the contralateral free vascularized fibular graft diameter. Then autologous bone grafts that were obtained from the iliac crest were tightly packed in the necrotic area with a custom-made tamp (Fig. 2).

\section{Free Vascularized Fibular Grafting}

The free vascularized fibular grafting was performed as described previously [10]. All patients underwent surgery by the same surgical team with experience in microsurgery. To reduce the operative time, preparation of the hip and harvesting of the fibular graft were performed concurrently by two teams.

\section{Postoperative Management}

Second-generation cephalosporins were administered twice a day for 3 days after surgery for prophylaxis. Prophylactic anticoagulation involved daily intravenous administration of low-molecular-weight dextran for 3 days and subcutaneous administration of low-molecular-weight heparin for 7 days. Gentle passive mobilization of the hip and knee began 7 days after surgery, while active mobilization was initiated 4 weeks postoperatively. The femoral head was protected from weightbearing for 3 months with patients using a wheelchair or two crutches; gradual weightbearing with the use of crutches was allowed after 3 months.

\section{Outcome Assessment}

\section{SPECT/CT Examination and Quantitative Analysis}

SPECT/CT examination was performed before surgery and at 6 and 36 months postoperatively, as previously described [33]. The data from SPECT/CT were analyzed by two experienced nuclear medicine physicians ( $\mathrm{YG}$ and $\mathrm{HY}$ ) who were not involved in the surgery or with group assignment. The SPECT images were processed with Astonish technology (Astonish Technologies-Philips, San Jose, CA, USA) and integrated with the CT image using SYNTEGRA $^{\mathrm{TM}}$ image fusion software (Precedence $^{\circledR}$ scanner; Philips, San Jose, CA, USA). The physicians continuously outlined the same-size region of interest (ROI) on both sides of the femoral head and the first sacral vertebra on all images; the ROI curve then was automatically generated on the SPECT/CT fusion images along with a three-dimensional reconstruction of the final image. The SYNTEGRA program calculates the radioactive nuclide counting of the ROI and expresses this calculation 
Fig. 2A-D Core decompression and bone grafting are shown in these images. (A) A custom-made hollow reamer was used to broach the tunnel until $0.5 \mathrm{~cm}$ below the subchondral bone using the $\mathrm{C}$-arm machine. (B) Necrotic bone was removed from the hollow reamer. (C) A custom-made curette was used to remove the rest of the necrotic bone along the tunnel. (D) Bone granules were tightly grafted to the necrotic area through a hollow reamer with a custom-made plugger.
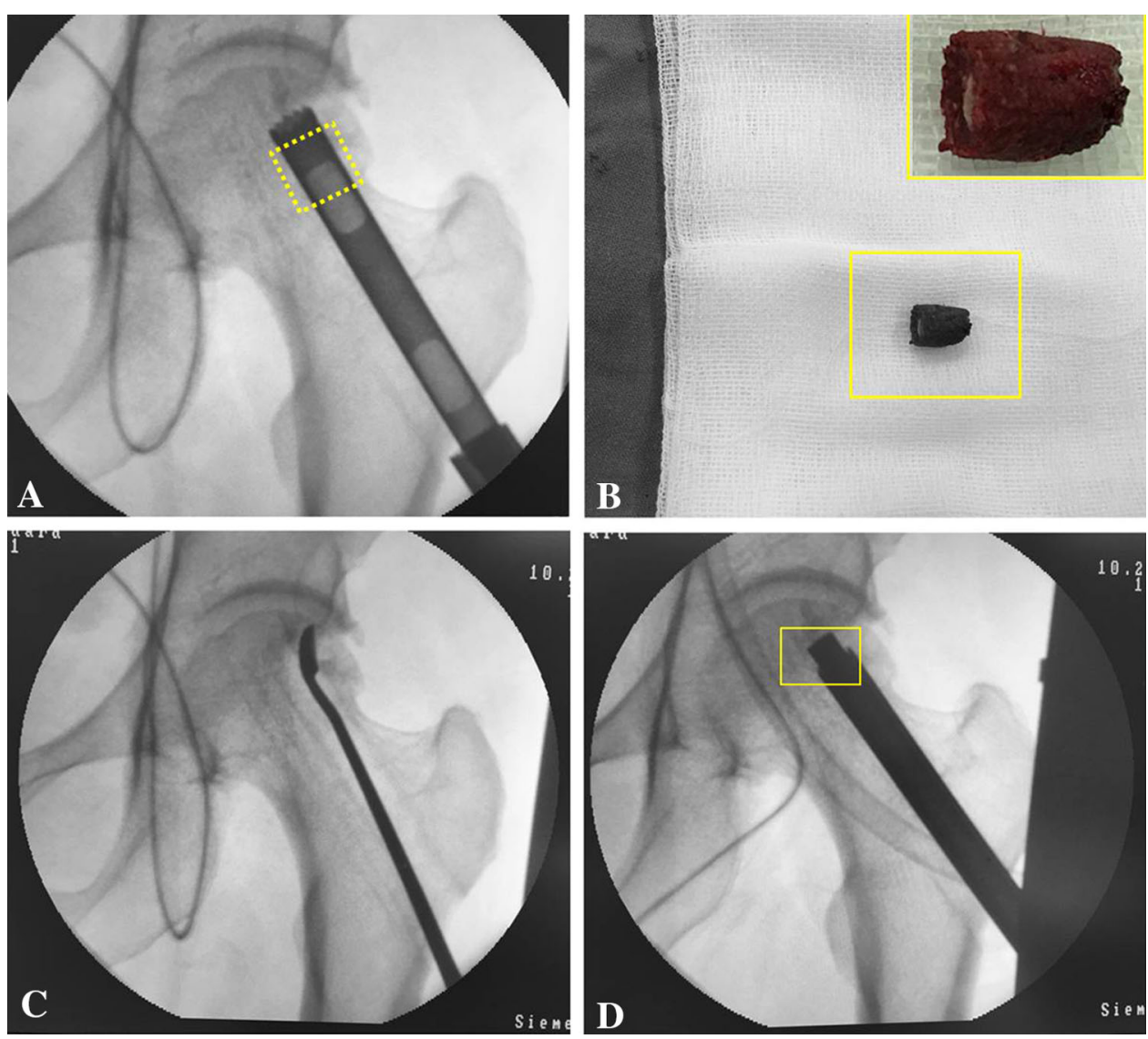

as $\gamma$ photons $/ \mathrm{cm}^{3}$. To minimize errors caused by radionuclide dose and the examining time, we used the $\gamma$ photon numbers per unit volume $\left(1 \mathrm{~cm}^{3}\right)$ ratio in the surgical site against the $\gamma$ photon numbers per unit volume in the first sacral vertebra to express the vascularity (V) status or blood supply of the femoral head.

$$
\begin{aligned}
\mathrm{Vcd}= & \left(\frac{\gamma \text { photonnumbers } / \mathrm{cm}^{3} \text { in the CD treated hip }}{\gamma \text { photonnumbers } / \mathrm{cm}^{3} \text { in the first sacral vertebra }}\right) \\
& \times 100(\%)
\end{aligned}
$$

$$
\begin{aligned}
\mathrm{Vf}= & \left(\frac{\gamma \text { photon numbers } / \mathrm{cm}^{3} \text { in the FVFG treated hip }}{\gamma \text { photonnumbers } / \mathrm{cm}^{3} \text { in the first sacral vertebra }}\right) \\
& \times 100(\%)
\end{aligned}
$$

where $\mathrm{CD}=$ core decompression and $\mathrm{FVFG}=$ vascularized fibular grafting. The vascularity status of the femoral head in decompression-treated hips before surgery and at 6 and 36 months after surgery was recorded as Vcd0, Vcd1, and Vcd2, respectively. The vascularity status of the femoral head in fibular-grafted hips before surgery and at 6 and 36 months after the operation was recorded as Vf0, Vf1, and Vf2, respectively.

\section{MRI Examination}

Outcomes also were evaluated with MR images that were obtained with a 1.5-T Siemens Aera ${ }^{\circledR}$ MRI scanner (Siemens Healthcare GmbH, Erlangen, Germany). Images of the hips were obtained before surgery and at $6,12,24$, and 36 months postoperatively. The MRI findings then were staged based on the ARCO classification.

\section{Clinical Outcomes}

All patients were assessed before treatment and 6, 12, 18, 24, 30 , and 36 months after the treatments; the assessment included plain film radiographs and HHS. Considering both hips of each patient were surgically treated at the same time, we recorded pain, limp, ROM, and deformity for each hip, and the function part of the HHS including walking distance, 
activities such as putting on shoes and socks, use of support such as a cane, ability to sit, use of stairs, and use of public transportation, were considered as a common score and added to the total HHS of each surgically treated hip.

Because it is difficult to differentiate the functional disability of the HHS from one hip to the contralateral hip in terms of walking distance, activities such as putting on shoes and socks, use of a support such as a cane, sitting ability, stairs, and use of public transportation, since those activities are shared by the more- and less-affected hips, the minimum clinically important difference (MCID) as estimated for the HHS will tend to be decreased in this study, which evaluated bilateral hips in the same patients. Others have identified a MCID of 16 to 18 points on the 100-point HHS [26]; because our between-group differences were likely to be depressed for the reason described, we defined a priori a difference of 10 points on the HHS to represent the MCID in this study.

Clinical failure was defined as deterioration of clinical symptoms that were severe enough to result in THA, and progression to THA was designated as the endpoint for followup.

\section{Statistical Analysis}

Hip survival was calculated via the Kaplan-Meier method using conversion to THA as an endpoint. The log-rank test was used to compare the survival rate between the two groups. Comparisons between the two groups were performed using a paired t-test (for continuous data) and Wilcoxon rank sum test (for nonparametric data). A probability less than 0.05 was considered statistically significant. All analyses were performed using $\mathrm{SAS}^{\circledR}$ software (SAS Institute Inc, Cary, NC, USA).

\section{Results}

\section{Vascularity Analysis on SPECT/CT}

We found that decompression-treated hips had lower vascularity as measured by SPECT/CT than fibular-grafted hips at 6 months $(68 \% \pm 6 \%$ versus $95 \% \pm 5 \%$; mean difference, $-27 \% ; 95 \% \mathrm{CI},-32 \%$ to $-23 \% ; \mathrm{p}<0.001)$ and 36 months $(57 \% \pm 4 \%$ versus $91 \% \pm 3 \%$; mean difference, $-34 \% ; 95 \%$ CI, $-37 \%$ to $-32 \%$; p < 0.001) (Fig. 3). SPECT/CT images show the differences seen in the ROI (Fig. 4).

\section{MRI Assessment During Followup}

The MRI results of the patients in the core decompression group showed that nine hips $(43 \%)$ progressed in ARCO stage after surgery, two (10\%) of which underwent subsequent THA. In the fibular-grafted group, one Stage II hip markedly progressed to Stage IV and required THA at 24 months after surgery. The distribution of ARCO stages in the core decompression group and fibular grafting group are shown (Table 3). Although the treatment groups were no different at 12 months (Stages I, II, III: 1/21, 11/21, 9/21 versus $2 / 21,13 / 21,6 / 21$, respectively; $\mathrm{p}=0.31$ ) and 24 months (Stages I, II, III: 0/21, 8/20, 12/20 versus 2/20, 12/ $20,6 / 20$, respectively; $p=0.06$ ) regarding progression of ARCO stage, progression was more severe in the core decompression group than the fibular-grafted group at 36
Fig. 3 SPECT/CT analyses at 6 months and 36 months after surgery show hips that had core decompression (CD) had lower vascularity than hips that had free vascularized fibular grafting (FVFG).

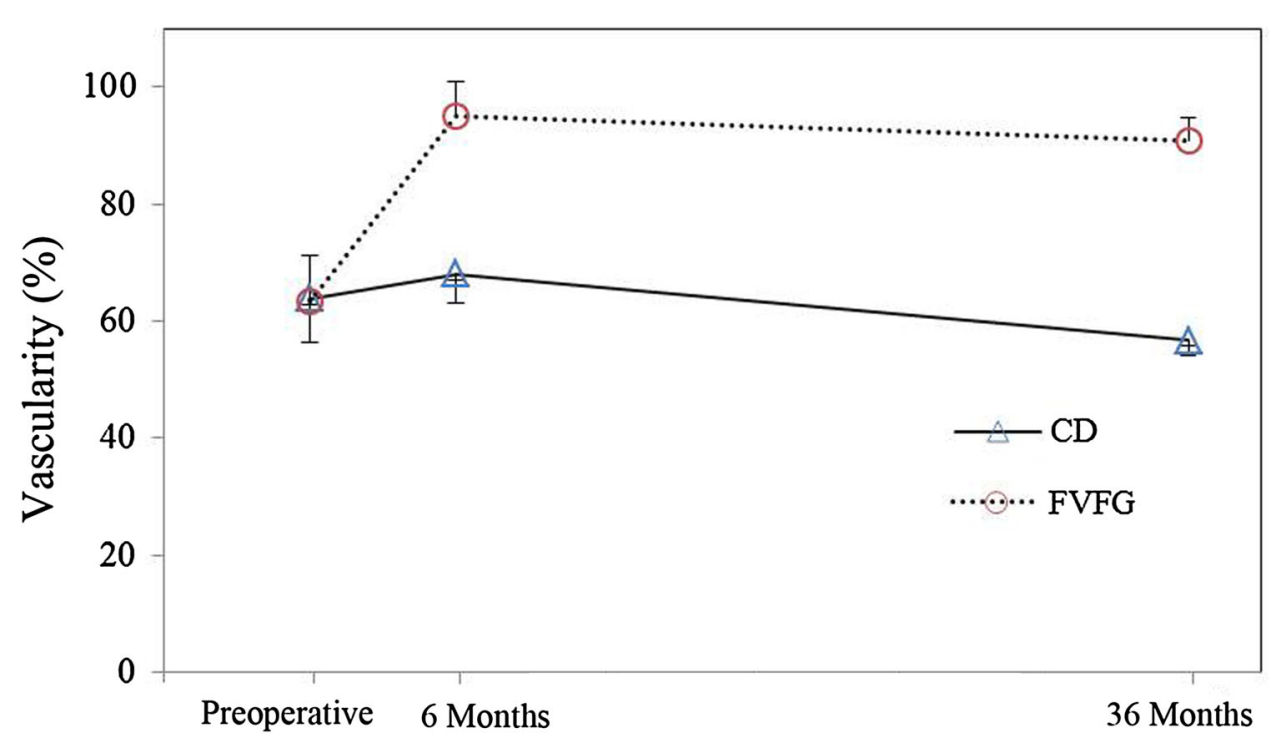



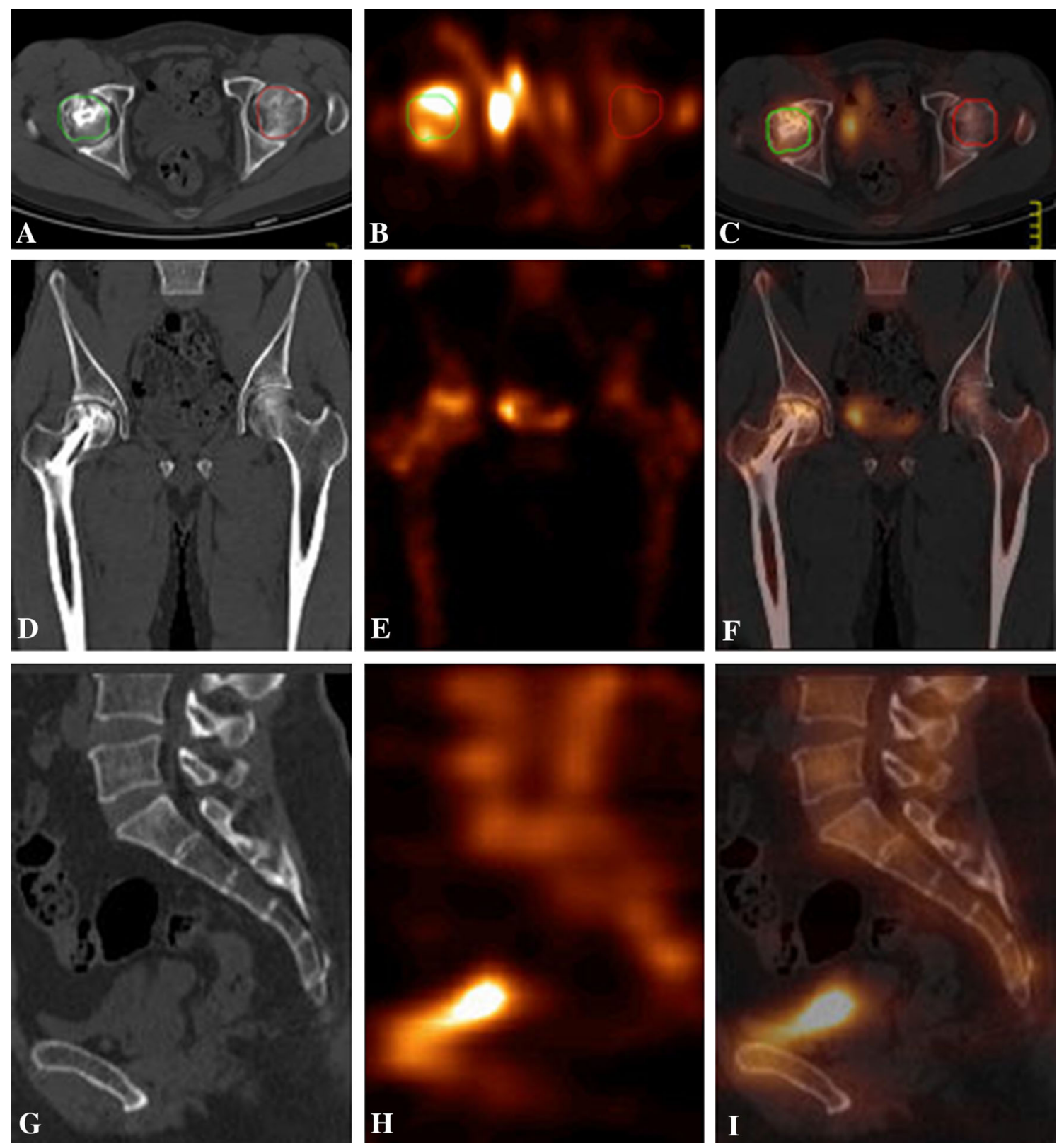

Fig. 4A-I The SPECT/CT images of the hips of a 43-year-old male patient are shown. The patient's left hip was treated by free vascularized fibular grafting and the right was treated with core decompression. (A) The region of interest was drawn around the femoral head on both femoral heads on the CT image. (B) The region of interest would automatically appear on SPECT and (C) fusion images of SPECT/CT. (D) The coronal CT image shows the patient's

months (Stages I, II, III: 0/19, 7/19, 12/19 versus 2/20, 12/ $20,6 / 20$, respectively; $p=0.03$ ).

\section{HHS and Progression to THA}

Core decompression-treated hips had lower HHS than vascularized fibular-grafted hips at every postoperative hips 36 months after surgery. (E) Radionuclide uptake of the patient's hips is shown in this SPECT image. (F) Vascularity of the femoral heads can be seen on the fused SPECT/CT image. (G) We used radionuclide uptake in the first sacral vertebra as a reference to minimize the errors caused by radionuclide dose. (H) The SPECT image shows radionuclide uptake in the first sacral vertebra. (I) A fused SPECT/CT image of the first sacral vertebra is shown.

time measured (Fig. 5); however, it was only after 18 months that the difference exceeded the MCID that we identified a priori of 10 points, meaning that the differences in favor of fibular grafting observed at earlier times were unlikely to have been clinically important to patients. The core decompression group had lower scores at 6 months (67 \pm 6 versus $76 \pm 5$; mean difference, -9 ; 95\% CI, -12 to $-6 ; \mathrm{p}<0.001), 12$ months $(71 \pm 6$ versus $81 \pm 3$; mean 
Table 3. Distribution of ARCO stages

\begin{tabular}{|c|c|c|c|c|c|c|c|c|c|c|}
\hline \multirow[t]{2}{*}{ ARCO stage } & \multicolumn{5}{|c|}{ Core decompression-treated hips } & \multicolumn{5}{|c|}{ Free vascularized fibular graft-treated hips } \\
\hline & Preoperative & 6 months & 12 months & 24 months & 36 months & Preoperative & 6 months & 12 months & 24 months & 36 months \\
\hline I & 3 & 3 & 1 & & & 2 & 2 & 2 & 2 & 2 \\
\hline II & 13 & 12 & 11 & 8 & 7 & 13 & 13 & 13 & 12 & 12 \\
\hline III & 5 & 6 & 9 & 12 & 12 & 6 & 6 & 6 & 6 & 6 \\
\hline \multicolumn{11}{|l|}{ IV } \\
\hline THA & & & & 1 & 2 & & & & 1 & 1 \\
\hline
\end{tabular}

$\mathrm{ARCO}=$ Association Research Circulation Osseous.

Fig. 5 Temporal trends for the Harris hip score showed that the mean score was lower in the core decompression (CD) group than in the free vascularized fibular graft (FVFG) group during the entire postoperative period.

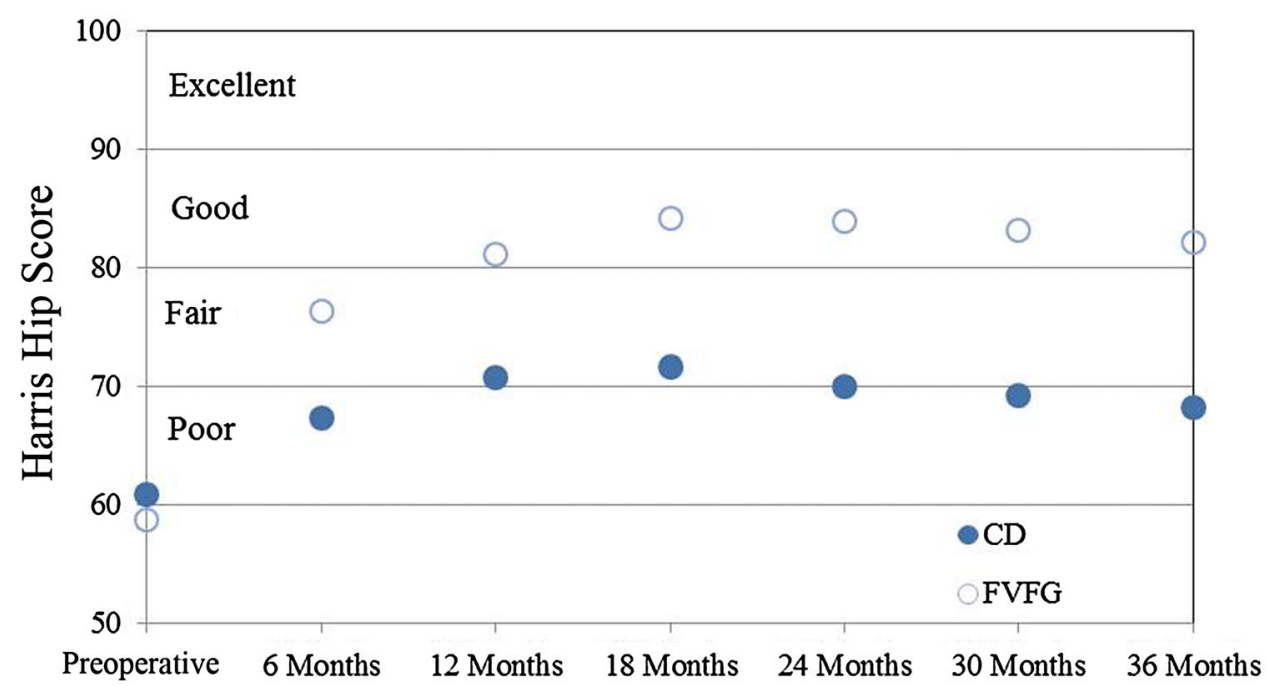

difference, $-10 ; 95 \% \mathrm{CI},-13$ to $-7 ; \mathrm{p}<0.001), 18$ months (72 \pm 4 versus $84 \pm 4$; mean difference, $-13 ; 95 \%$ CI, -15 to $-7 ; \mathrm{p}<0.001), 24$ months $(70 \pm 5$ versus $84 \pm$ 9; mean difference, -14 ; $95 \% \mathrm{CI},-17$ to -11 ; $\mathrm{p}<0.001)$, 30 months $(69 \pm 5$ versus $83 \pm 3$; mean difference, -14 ; $95 \% \mathrm{CI},-17$ to $-11 ; \mathrm{p}<0.001)$, and 36 months (68 \pm 5 versus $82 \pm 3$; mean difference, $-14 ; 95 \% \mathrm{CI},-17$ to $-11 ; \mathrm{p}<0.001)$. The differences in HHS mainly were attributable to the pain improvement.

At 24 months followup, one patient had progressed to having bilateral THAs. Another patient had the decompression-treated hip progress to THA at 30 months after surgery (Fig. 6). In the survival analysis, we found no difference between the two groups with conversion to THA as the endpoint at 36 months (two of 21 versus one of 21; log-rank test; $\mathrm{p}=0.893$ ).

\section{Discussion}

The goal in the treatment of osteonecrosis is to prevent head collapse and delay THA, especially in young individuals. However, the most-effective treatment for osteonecrosis continues to be debated [5, 13]. Maintenance of femoral head shape and the articular surface and halting progression of the disease appear to be among the preferred methods for avoiding arthrosis of the hip [5]. Modified core decompression plus implantation of bone progenitor cells has become one of the most-reliable and most-commonly used methods for treating osteonecrosis [11, 17, 21, 23, 27]. Although technically more complex, free vascularized fibular grafting is also a biologic option for hip preservation that may last 20 years or longer [1-3, 10, 31, 32]. It generally is accepted that vascularized fibular grafting is more likely to result in hip preservation by supporting the subchondral structure of the femoral head, reducing intraosseous pressure, removing and repairing necrotic tissue, and providing viable cortical and spongy bony structure in the treatment of osteonecrosis, which is especially preferred in young adults before radiographic collapse of the femoral head [10, 14]. Our randomized, controlled clinical trial was designed to evaluate the clinical outcome of core decompression and free vascularized fibular grafting in the treatment of osteonecrosis while also focusing on vascularity as shown by SPECT/CT and progression of ARCO stage based on followup MRI. 
Fig. 6 Survivorship curves for the hips in each treatment group, with conversion to THA as the endpoint are shown. Kaplan-Meier survivorship analysis showed no differences between the two groups in survival rates at 36 months after surgery. Log-rank test; $\mathrm{p}=$ 0.893; $\mathrm{CD}=$ core decompression; $F V F G=$ free vascularized fibular grafting.

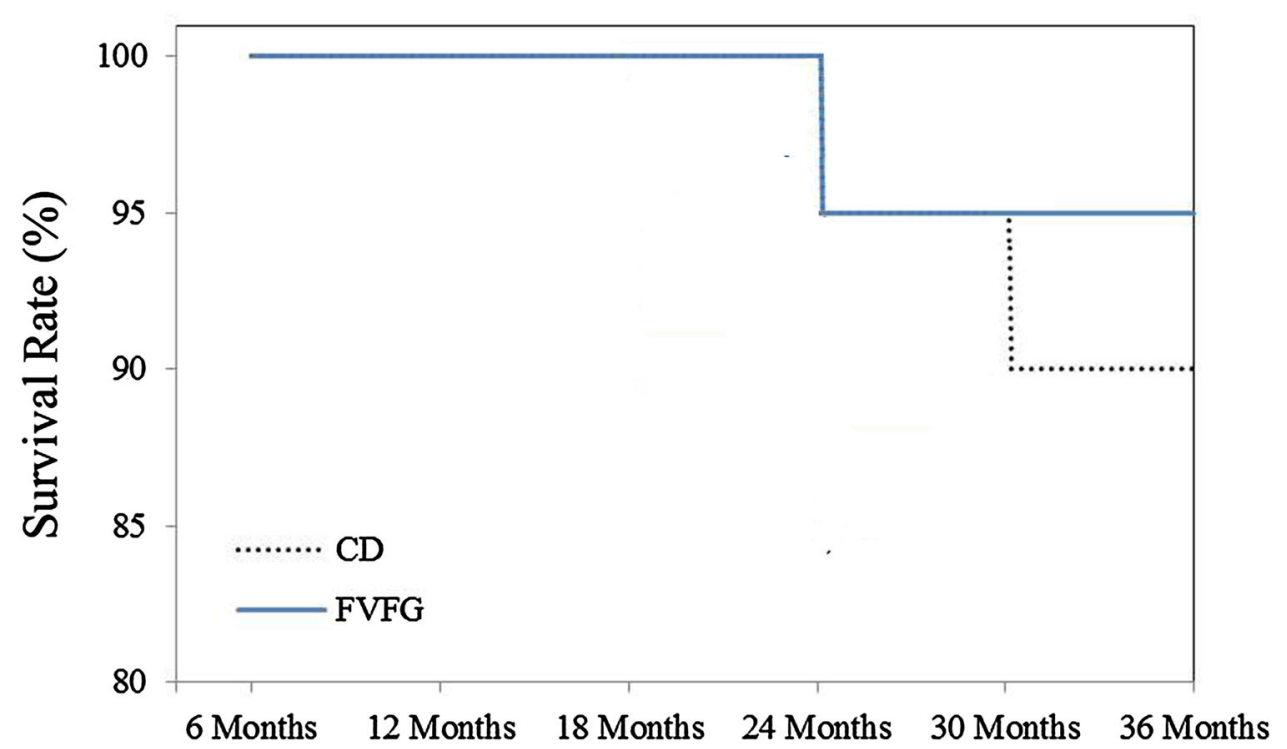

This study has numerous limitations. First, the number of patients in the study who had complete followup is relatively small. Six patients left the study owing to lack of SPECT/CT results or geographic relocation, which might have altered the outcomes and conclusions. The majority of the patients in our hospital are referred from other regions of China, which makes consistent followup difficult. Although our study is small, it has the advantage of comparing similar stages of necrosis in both hips of the same patient while examining the effects of two dissimilar procedures; therefore, we believe the number of patients is adequate for this type of study. Second, necrotic lesions of the femoral head were not analyzed. The focus of our study was vascularity of the femoral head and functional outcomes, which did not necessarily correlate with the MRI results. Evaluating bone graft healing and repair of the femoral head after surgery is difficult using two-dimensional MR images, which is why we elected to design a SPECT/CT study. The MRI analysis did not substantially contribute to our findings. Third, we assessed HHS per hip in the current study. It was very difficult to differentiate the functional disability of the HHS from one hip to the contralateral hip in terms of walking distance, activities such as putting on shoes and socks, use of a support such as a cane, sitting ability, stairs, and use of public transportation, since those activities are shared by the more- and lessaffected hips, so we used these same scores for each hip simultaneously. This would tend to depress the observed difference of the HHS. This also makes it difficult to use the published MCIDs of the HHS in the current study; others have identified a HHS of 16 to 18 as the MCID for that scoring tool [26]. Given that between-hip score differences were depressed here because function (walking distance, sitting ability, stairs) could not readily be distinguished between two hips in each patient, we defined a priori a difference of 10 points on the HHS to represent the MCID, but this is a best estimate given what is known; readers may reasonably consider it imprecise. Although the HHS of the fibular-grafted hips were better than those of core-decompressed hips, using the HHS we could not easily compare function between the hips for the reason described above; the differences we observed in the HHS were principally driven by pain. The long-term effect of improved vascularity of the femoral head and HHS still need to be defined; 3 years is probably not enough time to evaluate the durability of either procedure, given that the patients who undergo these procedures tend to be younger.

Our results showed that fibular-grafted hips had an increase in vascularity or blood supply of the femoral head compared with the core-decompressed hips. SPECT/CT analysis showed that vascularized fibular-grafted hips had higher vascularity than core-decompressed hips at 6 months and 36 months after surgery. The theoretical advantage of free vascularized fibular grafting is the inclusion of a new blood supply at the time of surgery, which allows for invasion of osteoinductive progenitor cells, revitalizing the once-necrotic zone, leading to restoration of a healthy subchondral plate [2]. Although comparison of nonvascularized and vascularized bone grafts has yielded supportive results $[12,22]$, improvement of blood flow to the femoral head after free vascularized fibular grafting is still poorly studied and lacks reliable imaging evidence. Zhao et al. [35] used digital subtraction angiography when selecting the vascularized bone graft for treatment of osteonecrosis. Digital subtraction angiography assessment was based on their experience in determining the circulation time and health of the femoral head. In 2016, Fontecha et al. [8] first reported femoral head bone 
viability of nine patients (10 hips) who underwent vascularized fibular grafting using SPECT/CT. They compared the fibular graft SPECT/CT signal intensity with the intensity at the ipsilateral proximal femoral diaphysis. A lower fibular graft intensity was given a score of 1 , equal intensity of both was given a score of 2 , and a higher fibular graft intensity was given a score of 3 . The SPECT/ CT findings revealed a progressive increase of femoral head uptake in all 10 hips, suggesting subchondral graft bone viability. In the current study, we performed SPECT/ CT to quantify the uptake of a radionuclide to assess vascularity of the femoral head after core decompression and free vascularized fibular grafting for treatment of bilateral osteonecrosis. Our results showed that vascularized fibulargrafted hips had an increase in vascularity or blood supply of the femoral head compared with the core decompression-treated hips, which supported the previous hypothesis concerning relevance of the vascular supply of the femoral head.

We found more progression to arthritis in the group treated with core decompression than the group treated with vascularized fibular grafting. Core decompression is one of the least-invasive femoral head-preserving procedures and is most effective during the early stages of osteonecrosis. This contrasts with the results of nonoperative treatment, wherein rates of femoral head preservation are low, and progression to collapse is rapid [16, 30]. This duration of preservation among patients with failed femoral head preservation compares favorably with results observed after core decompression. Scully et al. [24] compared 98 core decompression-treated hips (72 patients) with 614 free vascularized fibular-grafted hips (480 patients) with Ficat Stages I to III osteonecrosis. None of the 11 Stage I hips progressed to THA. The survival rate of fibular-grafted Stage II hips was 89\%, compared with $65 \%$ of decompression-treated hips at the same stage, at 50 months of followup [24]. Similarly, the survival rate of fibular-grafted Stage III hips was 50\%, compared with $21 \%$ of core-decompressed hips at this stage, at 50 months of followup [24]. Their result suggested that application of a vascularized fibular cortical strut to the subchondral bone may be superior to core decompression alone in terms of mechanical support and biologic enhancement of the necrotic femoral head.

Compared with the core decompression group, the increase in HHS observed in the fibular-grafted group mainly was attributable to pain improvement. Most of the patients showed apparent pain relief 6 months after surgery, which might be attributable to the improvement of vascularity of the femoral head. However, our betweengroup differences only reached the predefined MCID of 10 points (favoring fibular grafting) by 18 months after surgery. Given that the grafting procedure is much larger, it is perhaps not surprising that earlier HHS differences, which were mainly driven by pain, might not have been very great. We also note that at no point did the between-group difference in the HHS reach the 16 to 18-point MCID as defined by Singh et al. [26]; however, given that we evaluated bilateral hips in the same patients, the HHS in our study likely were depressed by the functionally worse of the two hips, and the MCID should likewise be considered smaller in a study of this design. We believe that a MCID of approximately 10 points in a study of our design to be appropriate, although we recognize this may be somewhat imprecise. We note, however, that scores greater than 80 on the HHS are considered good; reported HHS of fibular-grafted hips are frequently in excess of 80 $[7,10,22,29]$. Our mean HHS was 82 at the end of a 3year followup.

Hips treated with fibular grafting achieved greater vascularity, and by 3 years, improved ARCO staging. By 18 months after surgery, the hips treated with vascularized grafting also achieved a clinically important difference in terms of higher hip scores than those treated with core decompression, a difference that was certainly driven principally by pain relief (since functional differences are not distinguishable using the HHS in bilateral hips in the same patients). There were no between-group differences in terms of conversion to THA during this relatively short surveillance period. However, 3 years of followup may be too short, and future studies with longer-term followup are needed to determine whether fibular grafting can achieve sustained improvement in the vascularity of the femoral head, hip function, and avoidance of THA.

Acknowledgments We thank Yusen Gu MD and Haojun Yu MD (Department of Nuclear Medicine, Zhongshan Hospital, Fudan University) for assistance with SPECT/CT analysis, Teng Fei MD (Department of Orthopedic Surgery, Zhongshan Hospital, Fudan University) for assistance with the randomization sequence, and Fei Wang MD (Department of Biostatistics, Institute of Public Health, Fudan University) for assistance with the statistical analysis.

\section{References}

1. Aldridge JM 3rd, Berend KR, Gunneson EE, Urbaniak JR. Free vascularized fibular grafting for the treatment of postcollapse osteonecrosis of the femoral head: surgical technique. J Bone Joint Surg Am. 2004;86(suppl 1):87-101.

2. Aldridge JM 3rd, Urbaniak JR. Avascular necrosis of the femoral head: role of vascularized bone grafts. Orthop Clin North Am. 2007;38:13-22

3. Aldridge JM 3rd, Urbaniak JR. Vascularized fibular grafting for osteonecrosis of the femoral head with unusual indications. Clin Orthop Relat Res. 2008;466:1117-1124.

4. Bednarek A, Atras A, Gągała J, Kozak L. Operative technique and results of core decompression and filling with bone grafts in the treatment of osteonecrosis of the femoral head. Ortop Traumatol Rehabil. 2010;12:511-518. 
5. Chen CH, Chang JK, Lai KA, Hou SM, Chang CH, Wang GJ. Alendronate in the prevention of collapse of the femoral head in nontraumatic osteonecrosis: a two-year multicenter, prospective, randomized, double-blind, placebo-controlled study. Arthritis Rheum. 2012;64:1572-1578.

6. Dean GS, Kime RC, Fitch RD, Gunneson E, Urbaniak JR. Treatment of osteonecrosis in the hip of pediatric patients by free vascularized fibular graft. Clin Orthop Relat Res. 2001;386:106113.

7. Eward WC, Rineer CA, Urbaniak JR, Richard MJ, Ruch DS. The vascularized fibular graft in precollapse osteonecrosis: is longterm hip preservation possible? Clin Orthop Relat Res. 2012;470:2819-2826.

8. Fontecha CG, Roca I, Barber I, Menendez ME, Collado D, Mascarenhas VV, Barrera-Ochoa S, Soldado F. Femoral head bone viability after free vascularized fibular grafting for osteonecrosis: SPECT/CT study. Microsurgery. 2016;36:573577.

9. Gangji V, Hauzeur JP, Matos C, De Maertelaer V, Toungouz M, Lambermont M. Treatment of osteonecrosis of the femoral head with implantation of autologous bone-marrow cells: a pilot study. J Bone Joint Surg Am. 2004;86:1153-1160.

10. Gao YS, Chen SB, Jin DX, Sheng JG, Cheng XG, Zhang CQ. Modified surgical techniques of free vascularized fibular grafting for treatment of the osteonecrosis of femoral head: results from a series of 407 cases. Microsurgery. 2013;33:646-651.

11. Hernigou P, Beaujean F. Treatment of osteonecrosis with autologous bone marrow grafting. Clin Orthop Relat Res. 2002;405:14-23.

12. Kim SY, Kim YG, Kim PT, Ihn JC, Cho BC, Koo KH. Vascularized compared with nonvascularized fibular grafts for large osteonecrotic lesions of the femoral head. J Bone Joint Surg Am. 2005;87:2012-2018.

13. Korompilias AV, Beris AE, Lykissas MG, Kostas-Agnantis IP, Soucacos PN. Femoral head osteonecrosis: why choose free vascularized fibula grafting. Microsurgery. 2011;31:223-228.

14. Korompilias AV, Lykissas MG, Beris AE, Urbaniak JR, Souca$\cos$ PN. Vascularised fibular graft in the management of femoral head osteonecrosis: twenty years later. J Bone Joint Surg Br. 2009;91:287-293.

15. Lieberman JR, Engstrom SM, Meneghini RM, SooHoo NF. Which factors influence preservation of the osteonecrotic femoral head? Clin Orthop Relat Res. 2012;470:525-534.

16. Mankin HJ. Nontraumatic necrosis of bone (osteonecrosis). $N$ Engl J Med. 1992;326:1473-1479.

17. Mont MA, Hungerford DS. Non-traumatic avascular necrosis of the femoral head. J Bone Joint Surg Am. 1995;77:459-474.

18. Mont MA, Jones LC, Hungerford DS. Non-traumatic osteonecrosis of the femoral head: ten years later. J Bone Joint Surg Am. 2006;88:1117-1132.

19. Ohzono K, Saito M, Sugano N, Takaoka K, Ono K. The fate of nontraumatic avascular necrosis of the femoral head: a radiologic classification to formulate prognosis. Clin Orthop Relat Res. 1992;277:73-78.

20. Ohzono K, Saito M, Takaoka K, Ono K, Saito S, Nishina T, Kadowaki T. Natural history of nontraumatic avascular necrosis of the femoral head. J Bone Joint Surg Br. 1991;73:68-72.

21. Papakostidis C, Tosounidis TH, Jones E, Giannoudis PV. The role of "cell therapy" in osteonecrosis of the femoral head: a systematic review of the literature and meta-analysis of 7 studies. Acta Orthop. 2016;87:72-78.

22. Plakseychuk AY, Kim SY, Park BC, Varitimidis SE, Rubash HE, Sotereanos DG. Vascularized compared with nonvascularized fibular grafting for the treatment of osteonecrosis of the femoral head. J Bone Joint Surg Am. 2003;85:589-596.

23. Radke S, Rader C, Kenn W, Kirschner S, Walther M, Eulert J. Transient marrow edema syndrome of the hip: results after core decompression: a prospective MRI-controlled study in 22 patients. Arch Orthop Trauma Surg. 2003;123:223-227.

24. Scully SP, Aaron RK, Urbaniak JR. Survival analysis of hips treated with core decompression or vascularized fibular grafting because of avascular necrosis. $J$ Bone Joint Surg Am. 1998;80:1270-1275.

25. Shah SN, Kapoor CS, Jhaveri MR, Golwala PP, Patel S. Analysis of outcome of avascular necrosis of femoral head treated by core decompression and bone grafting. J Clin Orthop Trauma. 2015;6:160-166.

26. Singh JA, Schleck C, Harmsen S, Lewallen D. Clinically important improvement thresholds for Harris Hip Score and its ability to predict revision risk after primary total hip arthrplasty. BMC Musculoskelet Disord. 2016;17:256.

27. Tabatabaee RM, Saberi S, Parvizi J, Mortazavi SM, Farzan M. Combining concentrated autologous bone marrow stem cells injection with core decompression improves outcome for patients with early-stage osteonecrosis of the femoral head: a comparative study. J Arthroplasty. 2015;30(9 suppl):11-15.

28. Ünal MB, Cansü E, Parmaksızoğlu F, Çift H, Gürcan S. Treatment of osteonecrosis of the femoral head with free vascularized fibular grafting: results of 7.6-year follow-up. Acta Orthop Traumatol Turc. 2016;50:323-329.

29. Urbaniak JR, Coogan PG, Gunneson EB, Nunley JA. Treatment of osteonecrosis of the femoral head with free vascularized fibular grafting: a long-term follow-up study of one hundred and three hips. J Bone Joint Surg Am. 1995;77:681-694.

30. Vail TP, Covington DB. The incidence of osteonecrosis. In Urbaniak JR, Paul JP Jr, eds. Osteonecrosis: Etiology, Diagnosis, and Treatment. Rosemont, IL: American Academy of Orthopaedic Surgeons; 1997:43-49.

31. Wood MB. Free vascularized fibular grafting: 25 years' experience: tips, techniques, and pearls. Orthop Clin North Am. 2007;38:1-12.

32. Yoo MC, Kim KI, Hahn CS, Parvizi J. Long-term follow up of vascularized fibular grafting for femoral head necrosis. Clin Orthop Relat Res. 2008;466:1133-1140.

33. Yuan HF, Shen F, Zhang J, Shi HC, Gu YS, Yan ZQ. Predictive value of single photon emission computerized tomography and computerized tomography in osteonecrosis after femoral neck fracture: a prospective study. Int Orthop. 2015;39:1417-1422.

34. Zhao D, Cui D, Wang B, Tian F, Guo L, Yang L, Liu B, Yu X. Treatment of early stage osteonecrosis of the femoral head with autologous implantation of bone marrow-derived and cultured mesenchymal stem cells. Bone. 2012;50:325-330.

35. Zhao D, Xiaobing Y, Wang T, Wang B, Liu B, Fengde T, Fu W, Huang S, Qiu X. Digital subtraction angiography in selection of the vascularized greater trochanter bone grafting for treatment of osteonecrosis of femoral head. Microsurgery. 2013;33:656659. 